\title{
Peri-Operative Intracameral Antibiotics: The Perfect Storm?
}

\author{
Gary D. Novack ${ }^{1,2}$ and Jeffrey J. Caspar ${ }^{1}$
}

\begin{abstract}
In our University journal club we discussed a large, retrospective study of cataract surgery endophthalmitis rates before and after instituting the use of an intracameral fluoroquinolone antibiotic. We identified several factors involved in the use of off-label, compounded moxifloxacin in intraocular surgery. The introduction of phacoemulsification for cataract surgery led to the potential for smaller incisions. Intraocular lens technology improved to allow for foldable lenses, obviating the requirement to enlarge the incision. This allowed for clear corneal incisions, which unfortunately allow bidirectional passage of liquid. Preservatives were introduced into multidose ophthalmic products in the mid 20th century to retard microbial growth. However, more recently, chronic use of benzalkonium chloride has led to concerns about concerns about conjunctival toxicity, especially in patients with ocular surface disease. In the formulation of ocular moxifloxacin, developers were able to develop a "self-preserved", multi-dose product. Other concerns with eyedrops include varying levels of adherence and performance, and the expansion of compounding pharmacies from a named-patient basis to widespread national delivery, with concerns for lower quality. Integrating these factors, use of intracameral moxifloxacin as a prophylactic during cataract and other anterior segment surgery has become a standard of care in much of the U.S. We are concerned that the current position is on a narrow ledge-the standard of care for millions of surgeries each year based upon off-label, compounting use of a single product. We discuss possible ramifications and solutions to this public health issue.
\end{abstract}

Keywords: antibiotics, surgery, intracameral, prophylaxis

\section{Introduction}

W E PROVIDE A commentary of the multiple standard-ofcare, regulatory, and reimbursement issues for the offlabel use of intracameral antibiotics at the time of cataract surgery as prophylaxis for endophthalmitis. At a recent journal club, we discussed a very large, retrospective study of cataract surgery performed before and after instituting the use of an intracameral fluoroquinolone antibiotic. In this commentary, we present several factors that make our current status a "perfect storm" of a standard of care, which is inconsistent with approved therapy and evidence-based medicine.

\section{Journal Club Paper}

The article under discussion at our journal club evaluated patient complications of posterior capsular rupture, ${ }^{1}$ complementing a previous article with all surgeries. ${ }^{2}$ In the latter article, the incidence of postoperative endophthalmitis declined from $692(0.07 \%)$ of 993,009 eyes to $185(0.02 \%)$ of $1,069,634$ eyes $(P<0.001)$ with the use of intraocular antibiotics. In an accompanying editorial, Mamalis commented on the strengths (e.g., very large patient population and stratification of results based upon the postoperative complication) and weaknesses (sequential, rather than parallel study design). He further comments that a " ...barrier to the widespread adoption of routine intracameral antibiotic prophylaxis in the United States is the lack of an approved commercial formulation." 3 In our discussion, we identified the several factors involved in the use of off-label, compounded moxifloxacin in intraocular surgery.

\section{Phacoemulsification and Foldable Intraocular Lenses}

The introduction of phacoemulsification for cataract surgery by Kelman in $1967^{4}$ led to the potential for smaller

\footnotetext{
${ }^{1}$ Department of Ophthalmology \& Visual Sciences, University of California, Davis, California, USA.

${ }^{2}$ PharmaLogic Development, Inc., San Rafael, California, USA.
} 
incisions. Intraocular lens technology improved to allow for foldable lenses, obviating the requirement to enlarge the incision. The net effect of these 2 innovations was to allow for quickly constructed clear corneal incisions that eliminate the need for sutures. Although these changes were beneficial to both the surgeon and patient, bidirectional passage of liquid in clear corneal incisions was demonstrated by Taban et al. ${ }^{5}$ Thus, the potential for intraocular infection exists with some studies suggesting that the move from scleral to clear corneal incisions increased the risk for infectious endophthalmitis as much as 5 times. ${ }^{6,7}$

\section{Fluoroquinolones in Ophthalmology}

Topical fluoroquinolones were introduced into general ophthalmology practice in the 1990s and their then novel mechanism of action was viewed as a major therapeutic advantage. ${ }^{8,9}$ In addition to their U.S. approval for the treatment of bacterial conjunctivitis, some also received approval for the treatment of bacterial keratitis. Over the ensuing decades, new generations of fluoroquinolones were developed and approved, primarily for the indication of bacterial conjunctivitis.

\section{Preservatives in Ophthalmic Pharmaceuticals- Inclusion and Exclusion}

In the mid-20th century, there was a realization that solutions of ophthalmic medications in multidose bottles, left unrefrigerated, could grow microorganisms. This was especially true for fluorescein, a common diagnostic, which supported the growth of Pseudomonas aeruginosa. ${ }^{10} \mathrm{~A}$ number of preservatives were used in the formulation of marketed products. These included chlorbutanol, parabens, thimerosal, benzalkonium chloride (and related molecules), and polyquaternium-1. Safety concerns have attenuated the use of many of these agents, and most of today's preserved products use benzalkonium chloride and polyquaternium 1. However, chronic use of benzalkonium chloride has led to concerns about conjunctival toxicity, especially in patients with ocular surface disease or glaucoma requiring long-term use of ocular hypotensive agents. Benzalkonium chloride has also been long known to be toxic to the corneal endothelium. ${ }^{11}$

In the development of ocular moxifloxacin, the developers at Alcon were able to formulate a "self-preserved," multidose product. That is, the formulation met the requirements for sterility (21 CFR 200.50) ${ }^{12}$ without a preservative. Although the approved indications were for short-term use, nonetheless, this feature was viewed as a positive attribute in the competitive environment with other preserved 4th generation fluoroquinolones.

\section{Treatment Adherence and Performance}

Blackwell reviewed patient adherence in the 1970s. ${ }^{13}$ However, adherence was not well quantified. In the 1980s, Urquhart and colleagues concluded that while the pharmaceutical industry used a high level of quality evaluation in the manufacture, storage, and dispensation of drugs $(90 \%$ or greater), there was little evaluation of what happened once the drugs were in the patient's hands. For some systemic drugs, the measurement of therapeutic blood levels was undertaken, however, these are challenged by the "white coat effect," wherein patients are most likely to adhere just before a planned interaction with the health care system. Urquhart developed electronic monitors for recording patient administration. ${ }^{14-16}$ These were initially used in ophthalmology to objectively quantify patient adherence with the chronic ocular hypotensive medications pilocarpine (q.i.d.), timolol (b.i.d.), and latanoprost (q.d.) including the use of concomitant medications. ${ }^{17-19}$ Although some patients had good adherence, many did not, and ophthalmologists could not readily predict this behavior. ${ }^{20}$

\section{Drug Delivery Systems}

A better way to deliver ocular medications has been a goal for decades as well. ${ }^{21}$ Initial nonerodible ocular implants were approved in 1996 (ganciclovir) and 2005 (fluocinolone acetonide), and the first erodible implant approved in 2009 (dexamethasone). Just recently, 2 additional dexamethasone implants (2018) and a prostaglandin anterior chamber implant (2020) were approved. Thus, ophthalmologists are becoming more comfortable with these technologies, and selecting them as a method to minimize the need for patient adherence-literally taking the therapy out of the patient's hands.

\section{Compounding Pharmacies}

Compounding pharmacy is centuries old. Pharmacists have a close relationship with physicians, preparing formulations "as directed" on a patient-by-patient basis. In more recent times, such a relationship has been a beneficial and positive one for the patient, pharmacist, and ophthalmologist. For example, before marketed cyclosporine products were approved, pharmacists prepared topical ocular cyclosporine from the approved oral product in peanut or other oils. Ophthalmologists at our institution frequently prescribe, on a patient-by-patient basis, fortified antibiotics for the treatment of corneal ulcers and autologous serum for the treatment of ocular surface disease.

There has been a growth of compounding pharmacies who aliquot and compound medications on a larger scale (i.e., not only on a patient-by-patient basis), advertise on the Internet, and ship across interstate lines. Part of this is driven by the passage of the Drug Quality and Security Act (DQSA) in 2013. ${ }^{22}$ In 2019, the U.S. Food and Drug Administration (FDA) noted “...compounded drugs can serve an important role in meeting patients' medical needs that cannot be met by an FDA-approved drug..." "...Although compounded drugs can fill an important role for patients and the FDA recognizes the need to preserve access to these products, they may also present a greater risk to patients because, among other things, they are not required to undergo the agency's premarket review for safety, effectiveness and quality. These risks have become evident during inspections of outsourcing facilities when the agency has found concerning production practices that have resulted in recalls of compounded drug products and enforcement action against some firms." To that end, FDA set up a "Center of Excellence" to "provide in-person and online education and trainings; a conference to give outsourcing facilities, stakeholders and the agency the opportunity to exchange ideas and best practices; and market research to help inform the agency on key issues faced by 
outsourcing facilities." 23 As noted by Fiscella et al., "there can be health risks associated with compounded drugs that do not meet federal quality standards. Compounded drugs made using poor quality practices may be sub- or superpotent, contaminated, or otherwise adulterated. The risks associated with compounding drugs that must be sterile are particularly relevant in the ophthalmic space." There are several case reports of adverse events, including loss of vision with formulations of triamcinolone, moxifloxacin, and bevacizumab. ${ }^{24,25}$

\section{Standard of Care and Evidence-Based- Evaluation}

Integrating all these factors, our impression is that the current standard of care is (or is becoming) to use intracameral antibiotics, including those compounded from systemic dosage forms (vancomycin ${ }^{26}$ and cefuroxime ${ }^{7}$ ), and in particular, topical ophthalmic moxifloxacin as a prophylactic during cataract and other anterior segment surgery. In India, Haripriya et al. used a product manufactured for this purpose by an Indian firm. However, in the United States, this means using a product prepared from the marketed topical ocular product. This is clearly "off-label," as the package insert states "... solution should not be injected subconjunctivally, nor should it be introduced directly into the anterior chamber of the eye." In our institution (which includes several facilities at which surgery is performedsame-day surgery center, Veterans Administration facilities, etc.), and in our community colleagues, we found a diversity of practice. Some surgeons used branded moxifloxacin ophthalmic solution (Vigamox ${ }^{\circledR}$ ), and some used a generic product. Some used the medication straight from the multiuse bottle, others had it prepared by compounding pharmacies, and others diluted the product with balanced salt solution. Although most use moxifloxacin intracamerally, other surgeons also use it intravitreally with transscleral or transzonular injection as a compounded mixture of triamcinolone and moxifloxacin. This selection is based upon surgeon preference and availability at each institution. All are warned not to use a new solution form of preserved moxifloxacin $\left(\right.$ Mozexa $\left.{ }^{\circledR}\right)$, which is labeled “.... should not be injected subconjunctivally or introduced directly into the anterior chamber of the eye."

With the ground breaking European Society of Cataract \& Refractive Surgeons endophthalmitis prophylaxis study showing a fivefold risk reduction in endophthalmitis, ophthalmic surgeons have felt the need for the regular use of intracameral antibiotics. ${ }^{7}$ This was despite the large number of patients needed to treat to have a benefit, the potential costs and risks, and the lack of a parallel study with concurrent controls demonstrating efficacy. This informed the next question-what would it take to obtain prophylactic intracameral use on the label? We considered various clinical study designs-however, they would involve not using a standard of care in one of the treatment groups, and our group did not feel that this was ethical. We are aware that the American Society of Cataract and Refractive Surgeons is planning a study to evaluate this indication. However, as of this time, we are not aware of the design or status being public. Another possibility is the position of the FDA that they are open to use of Real World Evidence in drug approval. ${ }^{27}$ Recently, Pershing et al. used the American
Academy of Ophthalmology "IRIS Registry" to evaluate acute-onset endophthalmitis (within 30 days of surgery) in $8,542,838$ cataract surgeries performed in the United States between 2013 and 2017. They found an incidence of $0.04 \% .{ }^{28}$ It may be possible that such "Big Data" analyses might be useful with respect to antibiotic use.

Additional recent studies support the findings of Haripriya et al. For example, Moser et al. conducted a retrospective evaluation of 55,984 phacoemulsification surgeries in Spain over a 16-year period, 2002 to December 2017. In the period after the introduction of intracameral cefazolin (2004-2010), the cumulative incidence of endophthalmitis was $0.047 \%$, markedly lower than that in the previous period $(0.37 \%-0.47 \%)$. From January 2011 to December 2017, after the postoperative change of topical ofloxacin by moxifloxacin, the cumulative incidence dropped to $0.0037 \% .^{29}$ In a Cochrane review, Gower et al. concluded that "high-certainty evidence shows that injection with cefuroxime with or without topical levofloxacin lowers the chance of endophthalmitis after surgery, and there is moderate certainty evidence to suggest that using antibiotic eye drops in addition to antibiotic injection probably lowers the chance of endophthalmitis compared with using injections or eye drops alone." They went further to conclude that "...Clinical trials with rare outcomes require very large sample sizes and are quite costly to conduct; thus, it is unlikely that many additional clinical trials will be conducted to evaluate currently available prophylaxis.",26

\section{Conclusions}

We are concerned that the current position is on a narrow ledge - the standard of care for literally millions of surgeries each year in the United States is based upon off-label, compounding use of a single product. If for some reason that product was no longer available, an unrelated event limited its use, another preserved product was available (leading to potential confusion), the laws and regulations on compounding pharmacies changed, or the medical system stopped reimbursing for off-label use, then surgeons and patients would be in a very precarious position.

\section{Author Disclosure Statement}

Dr. Novack consults for numerous ophthalmic, pharmaceutical, and medical device firms. A complete listing of all of Dr. Novack's consulting affiliations can be found online. Dr. Caspar serves as a consultant for Alcon.

\section{Funding Information}

No funding was received for this article.

\section{Acknowledgment}

The authors acknowledge the scientific contribution of Edward Lee, M.D.

\section{References}

1. Haripriya, A., Chang, D.F., and Ravindran, R.D. Endophthalmitis reduction with intracameral moxifloxacin in eyes with and without surgical complications: results from 
2 million consecutive cataract surgeries. J. Cataract. Refract. Surg. 45:1226-1233, 2019.

2. Haripriya, A., Chang, D.F., and Ravindran, R.D. Endophthalmitis reduction with intracameral moxifloxacin prophylaxis: analysis of 600000 surgeries. Ophthalmology. 124:768-775, 2017.

3. Mamalis, N. Reducing the risk of endophthalmitis. J. Cataract. Refract. Surg. 45:1217-1218, 2019.

4. Kelman, C.D. In tune with the father of phacoemulsification. J. Cataract. Refrac.t Surg. 23:1128-1129, 1997.

5. Taban, M., Sarayba, M.A., Ignacio, T.S., Behrens, A., and McDonnell, P.J. Ingress of India ink into the anterior chamber through sutureless clear corneal cataract wounds. Arch. Ophthalmol. 123:643-648, 2005.

6. Holz, E.R. Reducing endophthalmitis risk factors: where we stand. Am. J. Ophthalmol. 139:1097-1098, 2005.

7. Endophthalmitis Study Group, European Society of Cataract \& Refractive Surgeons. Prophylaxis of postoperative endophthalmitis following cataract surgery: results of the ESCRS multicenter study and identification of risk factors. J. Cataract. Refract. Surg. 33:978-988, 2007.

8. Leibowitz, H.M. Clinical evaluation of ciprofloxacin $0.3 \%$ ophthalmic solution for treatment of bacterial keratitis. Am. J. Ophthalmol. 112 (Suppl):34S-47S, 1991.

9. Gwon, A. Topical ofloxacin compared with gentamicin in the treatment of external ocular infection. Ofloxacin Study Group. Br. J. Ophthalmol. 76:714-718, 1992.

10. Vaughan, D.G., Jr. The contamination of fluorescein solutions, with special reference to Pseudomonas aeruginosa (bacillus pyocyaneus). Trans. Pac. Coast Otoophthalmol. Soc. Annu. Meet. 34:137-149, 1953.

11. Gasset, A.R. Benzalkonium chloride toxicity to the human cornea. Am. J. Ophthalmol. 84:169-171, 1977.

12. Kaufman, B., and Novack, G.D. Compliance issues in manufacturing of drugs. Ocul. Surf. 1:80-85, 2003.

13. Blackwell, B. Treatment adherence. Br. J. Psychiatry. 129: 513-531, 1976.

14. Urquhart, J., and Chevalley, C. Impact of unrecognized dosing errors on the cost and effectiveness of pharmaceuticals. Drug Inform. J. 22:363-378, 1988.

15. Engstrom, F.W., and Urquhart, J. Electronic monitoring of medication compliance in depressed outpatients. Clin. Pharmacol. Ther. 45:159, 1989.

16. Blaschke, T.F., Osterberg, L., Vrijens, B., and Urquhart, J. Adherence to medications: insights arising from studies on the unreliable link between prescribed and actual drug dosing histories. Ann. Rev. Pharm. Toxicol. 52:275-301, 2011.

17. Kass, M.A., Gordon, M., Morley, R.E., Meltzer, D.W., and Goldberg, J.J. Compliance with topical timolol treatment. Am. J. Ophthalmol. 103:188-193, 1987.

18. Kass, M.A., Meltzer, D.W., Gordon, M., Cooper, D., and Goldberg, J. Compliance with topical pilocarpine treatment. Am. J. Ophthalmol. 101:515-523, 1986.

19. Robin, A.L., Novack, G.D., Covert, D.W., Crockett, R.S., and Marcic, T.S. Adherence in glaucoma: objective measurements of once-daily and adjunctive medication use. Am. J. Ophthalmol. 144:533-540, 2007.
20. Kass, M.A., Gordon, M., and Meltzer, D.W. Can ophthalmologists correctly identify patients defaulting from pilocarpine therapy? Am. J. Ophthalmol. 101:524-530, 1986.

21. Novack, G.D. Ophthalmic drug delivery: development and regulatory considerations. Clin. Pharmacol. Ther. 85: 539-543, 2009.

22. Anonymous. Drug Supply Chain Security Act (DSCSA), 2020 [12 March 2020]. Available from: https://www.fda.gov/drugs/ drug-supply-chain-integrity/drug-supply-chain-security-actdscsa. accessed June 10, 2020.

23. Anonymous. FDA takes efforts to improve quality of compounded drugs from outsourcing facilities through collaboration and education as part of new Center of Excellence, 2020. Available from: https://www.fda.gov/newsevents/press-announcements/fda-takes-efforts-improvequality-compounded-drugs-outsourcing-facilities-throughcollaboration-and. accessed June 10, 2020.

24. Fiscella, R., Leiter, C., Massomi, F., Noecker, R., and Sheybani, A. Adjunctive antifibrotic therapy with mitomycin $\mathrm{C}$ and 5-fluorouracil: a review of their use in glaucoma surgery and considerations around ophthalmic compounding. Curr. Trends Ophthalmol. 2:154-169, 2019.

25. Anonymous. U.S. Illnesses and Deaths Associated With Compounded or Repackaged Medications 2001-19. 2020. Available from: https://www.pewtrusts.org/en/researchand-analysis/data-visualizations/2020/us-illnesses-and-deathsassociated-with-compounded-or-repackaged-medications2001-19 accessed June 10, 2020.

26. Gower, E.W., Lindsley, K., Tulenko, S.E., Nanji, A.A., Leyngold, I., Mcand Donnell, P.J. Perioperative antibiotics for prevention of acute endophthalmitis after cataract surgery. Cochrane Database Syst. Rev. 2:CD006364, 2017.

27. Novack, G.D. Pipeline: real world evidence for pharmaceuticals. Ocul. Surf. 17:584-588, 2019.

28. Pershing, S., Lum, F., Hsu, S., et al. Endophthalmitis after cataract surgery in the United States: a report from the intelligent research in sight registry, 2013-2017. Ophthalmology. 127:151-158, 2020.

29. Moser, C.L., Lecumberri Lopez, M., Garat, M., and MartinBaranera, M. Prophylactic intracameral cefazolin and postoperative topical moxifloxacin after cataract surgery: endophthalmitis risk reduction and safety results in a 16year study. Graefes Arch. Clin. Exp. Ophthalmol. 257: 2185-2191, 2019.

Received: March 24, 2020

Accepted: May 24, 2020

Address correspondence to: Dr. Gary D. Novack PharmaLogic Development, Inc. 17 Bridgegate Drive San Rafael, CA 94903 USA

E-mail: gary_novack@pharmalogic.com 